Article

\title{
Control Strategy Based on the Flexible Multi-State Switch for Maximum Consumption of Distributed Generations in Distribution Network
}

\author{
Guopeng Zhao ${ }^{1, * \mathbb{D}}$, Jiaxing Liu ${ }^{1}$, Siyuan Liu ${ }^{1}$, Xinwei Zhou ${ }^{1}$, Jihong $\mathrm{Li}^{2}$ and $\mathrm{Yi} \mathrm{Lu}^{3}$ \\ 1 School of Electrical and Electronic Engineering, North China Electric Power University, Beijing 102206, China \\ 2 State Grid Zhejiang Electric Power Co., Ltd, Hang Zhou 310027, China \\ 3 State Grid Zhejiang Electric Power CO., LTD. Research Institute, Hang Zhou 310014, China \\ * Correspondence: zhaoguopeng@ncepu.edu.cn; Tel.: +86-010-61771713
}

Received: 27 June 2019; Accepted: 15 July 2019; Published: 18 July 2019

\begin{abstract}
With the aim of improving the consumption capacity of distributed generation (DG) in the distribution network, the control strategy of the flexible multi-state switch (FMSS) for the maximum consumption of distributed generation is proposed. The principle that FMSS improves the consumption capacity of distributed generation is analyzed and verified by simulation. The estimating method for the maximum accessible capacity of distributed generation at the access point is proposed. For complex systems, the multi-objective function for maximizing the consumption of distributed generation was established, and the analytic hierarchy process and entropy weight method were combined to obtain the weight factor. Then, combined with system constraints, the results can be obtained by optimization algorithms. Finally, the control strategy of FMSS for maximum consumption of distributed generation was realized and verified in a simulation.
\end{abstract}

Keywords: flexible multi-state switch; consumption of distributed generation; analytic hierarchy process; distribution network

\section{Introduction}

With the increasingly prominent energy crisis, environmental degradation and other problems, distributed generation (DG) as an environmentally friendly, efficient and flexible form of power generation has been widely investigated and developed. Due to its inflexible structure and limited regulation means, the traditional distribution network cannot effectively suppress the uneven distribution of power flow brought by the access of DG, and the over-limit of feeder voltage, which limits the consumption capacity of DG in the distribution network [1-3].

At present, there are two kinds of research methods on how to improve the consumption capacity of DG in the distribution network. One is to improve the consumption of DG by using the regulation method in the traditional distribution network. Some studies [4-6] introduced the distribution network reconstruction method oriented to the consumption of DG to obtain the optimal distribution network reconfiguration results and DG output scheme. However, the reconfiguration of the distribution network is completed by the opening and closing of the contact switch. In the actual operation process, there are problems, such as the delay of switching operations and the impact of the closing ring current, which affect the safe and reliable operation of the network. A further study [7] adopted a distributed model for predictive control to optimize and control the controllable DG, energy storage, flexible load and other controllable resources in the region. However, the optimization results depend on the accuracy of the model's prediction and cannot guarantee the actual optimization results.

In recent years, with the development of power electronics technology, the use of flexible devices to improve the ability of distribution network to consume DG has become the main research direction. 
Among them, these studies $[8,9]$ changed the reactive power flow and adjusted the voltage deviation of the distribution network to increase the output of the distributed power supply by optimizing the configuration mode of the static var compensator (SVC) and static synchronous compensator (STATCOM) respectively. However, these methods can only adjust the distribution of reactive power flows. Therefore, the consumption ability of the distributed power supply needs to be improved.

Compared with the traditional power electronic reactive power compensation device mentioned above, a flexible multi-state switching (FMSS) device is featured with decoupling control of the power and reactive power, continuously adjustable power and fast response speed. This is the premise to realize the consumption capacity of DG in an optimized distribution network [10].

However, some studies [11,12] mainly realized the load transfer and network frame reconstruction between feeders of the distribution network through a soft-normally-open point (SNOP), to improve the consumption capacity of DG in the distribution network. Further studies [13] compared the consuming capacity of the three-terminal SNOP with a two-terminal SNOP for distributed power supply, and concluded that the three-terminal SNOP consumed 20\% more distributed energy than the two-terminal SNOP, which verified the superiority of a multi-terminal flexible switch compared to a two-terminal flexible switch. Another study [14] established the flexible distribution of the distributed power maximum access capacity model, the multiple population genetic algorithm to obtain the global optimal solution and analysis, and completed the flexible distribution of DG based on SNOP maximum access capacity calculations. The maximum consumable capacity of a distributed power supply with different SNOP access modes and capacities is analyzed in detail.

However, the methods mentioned above all laid particular emphasis on system planning and topological structure change, and focused on how to use FMSS to improve the capacity of the distribution network to consume DG. There was no detailed study on the operation control strategy of FMSS, and therefore, real-time regulation of DG was not possible.

There are relatively few studies on the operation control strategy of DG based on FMSS. Further studies $[15,16]$ provided a detailed comparative analysis of network reconfiguration with SNOP, verified the advantages and potential benefits of SNOP in real-time adjustment, and put forward a simulated annealing (SA) and conic programming $(\mathrm{CP})$ of hybrid optimization algorithms. This was to achieve the purpose of the distribution network operation sequence optimization. However, its objective function is the minimum value of energy loss in a period of an operation cycle, that is, $24 \mathrm{~h}$ to obtain a set of optimization results, which obviously does not meet the control requirements of real-time operation of the system.

Another study [17] established a two-stage tunable robust optimization model, which improved the calculation accuracy and efficiency of the robust optimization model, generated the robust operation strategy of the photovoltaic system, and improved the consumption capacity of the distribution network to photovoltaic. However, the voltage index in the objective function only works when the voltage exceeds the limit. Therefore, in the process of real-time adjustment of the system, the over-limit voltage may occur. In a further study [18], a sensitivity method based on a Jacobian matrix was proposed to determine the adjustable capacity boundary of SNOP when the grid/feeder at both ends of SNOP had different loads and power generation conditions to obtain its control strategy. However, this research is still focused on the level of distribution network planning, and its method has the potential of real-time operation control, but still needs to be optimized and improved.

In this paper, a strategy regulation of FMSS is proposed to maximize the consumption of DG in the complex distribution network. Firstly, the principle of FMSS promoting the consumption of DG is analyzed. Secondly, the related indexes and constraints of the distribution network's consumption of DG are analyzed. The supply system operation constraints for the distributed power's given situation and voltage fluctuation index constructs the objective function using the improved particle swarm optimization algorithm. From this, the instruction value of each port of the flexible multi-state switch and the maximum output value of DG are obtained. Finally, a complex network distribution system 
based on three IEEE-33 node networks is used to verify the correctness and superiority of the real-time control strategy of FMSS for maximum consumption of DG.

\section{Analysis of the Principle of FMSS Promoting the Consumption of DG}

\subsection{Analysis of the Capacity of Traditional Distribution Network to Consume DG}

When analyzing the consumption capacity of the distribution network DG, the permitted access capacity of DG is firstly analyzed on the premise that the voltage of the access point of DG does not exceed the limit [19]. In this paper, the DG is equivalent to a special load with negative power. In the calculation of the system power flow distribution and voltage analysis, the access node of DG can be equivalent to $\mathrm{PQ}$ node. A method based on the short-circuit capacity of the access point can be used to determine the maximum access capacity of DG.

The equivalent circuit of Thevenin of Figure 1 is an example for analysis. $E$ is the power supply voltage of the equivalent circuit, $Z_{S}$ is the equivalent impedance between the access point and the system power supply, and $U$ is the voltage at the access point of DG. Assuming the corresponding current change on the line caused by the output of DG is $\Delta I$, the voltage change of the access point value as shown in (1).

$$
\Delta U=(R+j X)\left(\Delta I_{p}+j \Delta I_{q}\right)=\left|Z_{s}\right||\Delta I|[(\cos \phi \cos \theta-\sin \phi \sin \theta)+j(\sin \phi \cos \theta+\cos \phi \sin \theta)]
$$

where $\phi$ is the impedance angle, $\theta$ is the angle of the DG output current leading the voltage, that is, the negative value of the power factor angle.

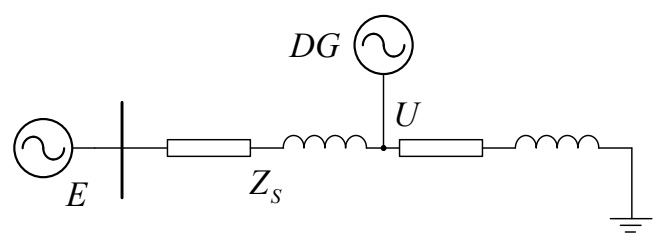

Figure 1. Thevenin equivalent circuit of the distributed generation (DG) access point.

The short-circuit capacity of the DG access point is calculated as shown in (2)

$$
S_{k}=\frac{U^{2}}{\left|Z_{S}\right|}
$$

where $S_{k}$ is the short circuit capacity of the distributed power supply access point. $Z_{S}$ is the equivalent impedance between the access point and the system power supply, and $U$ is the voltage at the access point of DG.

Combined with Equations (1) and (2), and as the distribution network analyzed in this paper is of voltage grade below $110 \mathrm{kV}$, the phase displacement at both ends of the line is not large. Therefore, the lateral component of the voltage variation can be ignored, thus, the relationship between the voltage variation of the access point of DG and the short-circuit capacity of the access point can be obtained as shown in (3):

$$
\Delta U=\frac{U^{2}}{S_{\mathrm{K}}} \frac{\left|\Delta S_{\mathrm{n}}\right|}{U}(\cos \phi \cos \theta-\sin \phi \sin \theta)=\frac{U^{2}}{S_{\mathrm{K}}} \frac{\left|\Delta S_{\mathrm{n}}\right|}{U} \cos (\phi+\theta)
$$

where $\Delta S_{\mathrm{n}}$ is DG access apparent power size, namely $\Delta S_{\mathrm{n}}=P_{\mathrm{DG}}+j Q_{\mathrm{DG}}$.

Therefore, the relationship between the voltage deviation of the access point and access capacity of DG is:

$$
d \%=\frac{\Delta U}{U}=\frac{\left|\Delta S_{\mathrm{n}}\right| \cos (\phi+\theta)}{S_{\mathrm{K}}} \times 100 \%
$$


According to Equation (4), when the allowable upper limit $d_{\max }$ of distribution network voltage deviation is known, the maximum allowable access capacity of DG at this access location can be obtained according to Equation (4), as shown in (5).

$$
S_{\mathrm{DGmax}} \approx \frac{S_{\mathrm{K}} d_{\max } \%}{100 \cos (\phi+\theta)}(\cos \theta+\mathrm{j} \sin \theta)
$$

For the traditional distribution network, the maximum capacity of the accessible DG depends on the maximum allowable voltage deviation of the distribution network. This occurs when the impedance angle between the power supply and the access point of DG, and the power factor of the access of DG and the short-circuit capacity at the input node have been determined.

\subsection{Analysis of Consumption Capacity of DG after FMSS Is Connected}

With the continuous increase of the permeability of DG, it is difficult to regulate the power flow of the traditional distribution network by traditional means. The power flow of each branch may exceed the limit of the feeder power flow, and the voltage of each node in the system can also greatly deviate from the nominal voltage of the system and exceed the allowable value of the voltage deviation. By virtue of its flexible and fast power flow regulation ability, FMSS optimizes the power flow distribution under the condition of high permeability of DG and inhibits the off-limit of the branch power flow and node voltage deviation to improve the consumption ability of DG in the distribution network.

The following is an example of the simple double-feeder distribution network model shown in Figure 2 to introduce the principle of FMSS to improve the consumption capacity of DG in the distribution network.

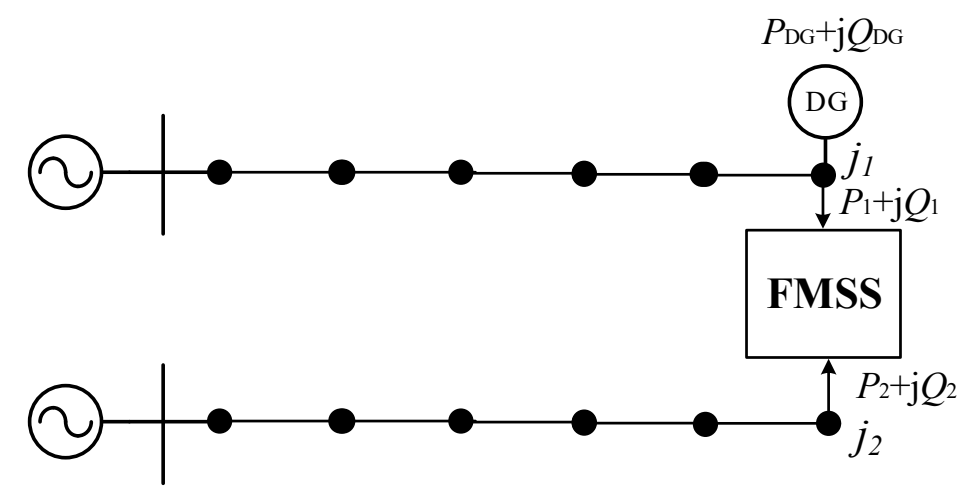

Figure 2. Simple uniform distribution network system with DG.

In the simple distribution network shown in Figure 2, the two feeder ends are connected by FMSS. According to the operating constraints of FMSS, the active and reactive power relationship of the two ports is shown in (6).

$$
\left\{\begin{array}{c}
P_{1}+P_{2}=0 \\
-\sqrt{S_{1}^{2}-P_{1}^{2}} \leq Q_{1} \leq \sqrt{S_{1}^{2}-P_{1}^{2}} \\
-\sqrt{S_{2}^{2}-P_{2}^{2}} \leq Q_{2} \leq \sqrt{S_{2}^{2}-P_{2}^{2}}
\end{array}\right.
$$

The access DG is at $j_{1}$, end node of feeder 1 . According to (5), the maximum capacity of DG that node $j$ can access is $S_{D G \text { max }}=P_{D G}+j Q_{D G}$. At this point, the node voltage deviation is shown in (7). If the DG access capacity exceeds this value, the voltage deviation of node $j_{1}$ exceeds the allowable voltage offset value.

$$
\Delta U_{j 1 \max }=\Delta U_{j 1}=\frac{\left(P_{\mathrm{DG}}-P_{j 1}\right) R+\left(Q_{\mathrm{DG}}-Q_{j 1}\right) X}{U_{\mathrm{N}}}>0
$$


when FMSS is connected, the voltage at node $j_{1}$ changes to:

$$
\Delta U_{j 1}^{\prime}=\frac{\left(P_{\mathrm{DG}}-P_{1}-P_{j 1}\right) R+\left(Q_{\mathrm{DG}}-Q_{1}-Q_{j 1}\right) X}{U_{\mathrm{N}}} \leq \Delta U_{j 1 \max }
$$

Meanwhile, the voltage change at the end node $j_{2}$ on feeder 2 is:

$$
\Delta U_{j 2}^{\prime}=\frac{\left(-P_{2}-P_{j 2}\right) R+\left(-Q_{2}-Q_{j 2}\right) X}{U_{N}}=\frac{\left(P_{1}-P_{j 2}\right) R+\left(-Q_{2}-Q_{j 2}\right) X}{U_{N}}
$$

In the low-voltage distribution network, there is little difference between the resistance and inductance, and the influence of resistance cannot be ignored. Therefore, both active power and reactive power have some influence on the voltage offset. By type (8), when FMSS absorbs active power from feeder 1 and transfers it to the end of feeder 2, the node voltage offset caused by the active power of DG at node $j_{1}$ can reduce. If FMSS simultaneously absorbs inductive power from feeder 1 , the voltage offset caused by DG can further reduce, therefore, the digestible DG capacity at node $j_{1}$ increases. At the same time, Equation (9) shows that since feeder 2 receives the active power transferred from feeder 1 by FMSS, the terminal node voltage increases and the offset also decreases. If FMSS absorbs capacitive reactive power here, the terminal node voltage will further increase. It can be seen that FMSS can increase the consumption capacity of distribution network to the DG by regulating the active power flow between feeders and the reactive power at the port, and at the same time, achieve the effect of feed load balance and overall reduction of node voltage deviation.

\section{The Control Strategy of FMSS for Maximum Consumption of DG}

The above analysis of the consuming ability of DG in the distribution network after FMSS is connected illustrates the principle of FMSS promoting the consuming ability of DG in the distribution network. In this section, for large-scale systems, the DG that can be absorbed by each node under the rated capacity of FMSS can be solved through optimization algorithms after multiple nodes are connected to DG. Therefore, the FMSS real-time control strategy aiming at the maximum consumption of DG is realized.

\subsection{The Objective Function for Consumption of DG Is Established}

\subsubsection{DG Maximizes Consumption Indexes}

The maximum consumption of DG refers to the maximum active power output of DG that can be absorbed by the distribution network under the constraints of system operation. Its value is mainly related to the distribution network structure, the system operation mode, the access mode, the access location and the access power factors of DG [20]. According to the theoretical analysis in the first part of this paper, the main factor limiting the consumption of DG is the maximum allowable value of voltage deviation. Therefore, for the relevant evaluation indexes of the maximum consumption of DG in the distribution network, this paper mainly considers two indexes, namely, the output of DG and voltage deviation. The calculation method of indexes is shown in (10) and (11).

(1) The output of DG

$$
\min f_{1}=\sum_{i \in N_{D G}}\left(P_{D G . i \max }-P_{D G . i}\right)
$$

where $P_{\mathrm{DG} . i m a x}$ is the maximum active power output by DG; $P_{\mathrm{DG} . i}$ is the actual active power output of DG; and $N_{\mathrm{DG}}$ is the number set of DG.

(2) Voltage deviation:

$$
\min f_{2}=\sum_{t=1}^{M} \sum_{n=1}^{N_{t}}\left|\left(U_{t, n}\right)^{2}-1\right|
$$


In this paper, the advantages of using the above two indicators as the objective function is, in sampling time, to take all network node voltages and all of the DG output as optimization objectives in each sampling instant. Therefore, at every sampling moment, the voltage quality and DG output of the whole distribution network can be optimized to achieve a real-time operation effect. Compared with the objective function in references $[15,16]$ mentioned above, it has better real-time characteristics.

Where, $t$ is the network number, $n$ is the number of nodes, $U_{t, n}$ is the voltage per unit value of $n$ nodes in network $t, U_{t}$ is the node set in each network, and $M$ represents the number of network interconnection based on multi-terminal flexible DC devices.

Considering the above two operating indexes, the optimization problem is transformed into a multi-objective optimization form. The multi-objective optimization problem cannot simply determine the optimal solution by comparing the value of the objective function. The linear weighted sum method is usually used to convert multiple targets into a single target, as shown in (12):

$$
\min f=\lambda_{1} f_{1}+\lambda_{2} f_{2}
$$

where $\lambda$ is the weight coefficient, $\lambda_{1}+\lambda_{2}=1$.

\subsubsection{Objective Function Weight Coefficient Selection Method}

This paper combines the analytic hierarchy process and the entropy weight method to determine the index weight of the objective function in Equation (12).

The analytic hierarchy process (AHP) is a multi-objective optimal weight coefficient allocation method that combines qualitative and quantitative analysis. Its advantage is that it can quantify the experienced judgments of decision makers, which is more practical in the case of complex target structures and the lack of necessary data [21]. The entropy weight method is an objective method to judge the weight coefficient of each index, and determine the weight of each index according to the variability of the index, and can make the value of each index into a dimensionless form.

As AHP is based on expert experience and subjective scores, the influence of the expert's rich experiences on index weight cannot be ignored. However, the entropy weight method is an objective evaluation method, which calculates the weight according to the objective data, reflects the intensity of relative competition of the index data, and its influence on index weight cannot be ignored. Therefore, the combination of the analytic hierarchy process and the entropy weight method can make the final weight coefficient consider both subjective and objective factors, and unify the dimensions of each sub-function when determining the weight coefficient in this paper.

Assuming the weight factor obtained by the analytic hierarchy process is $\omega_{j}$ and the weight factor obtained by the entropy weight method is $k_{j}$, then the calculation method of the comprehensive weight coefficient determined by combining the analytic hierarchy process and the entropy weight method is shown in (13).

$$
\lambda_{j}=\frac{\omega_{j} k_{j}}{\sum_{j}^{m} \omega_{j} k_{j}}
$$

\subsection{Constraints of Distribution Network Operation Including Flexible Multi-State Switch}

\subsubsection{Operating Constraints of Flexible Multistate Switches}

This paper takes FMSS of three ports as an example to study the constraint conditions of power flow control of each port when FMSS is in a steady state operation. The topology of the three-terminal flexible multi-state switch is shown in Figure 3. Where, $A$ end and $B$ end are running in the rectifier state, and $C$ end is running in the inverter state. $Z_{A}, Z_{B}$ and $Z_{C}$ are the ac side impedance at the three ends of the device. In general, the rated operating loss of the flexible multi-state switches is approximately $3 \%-5 \%$ of the rated power [22]. 


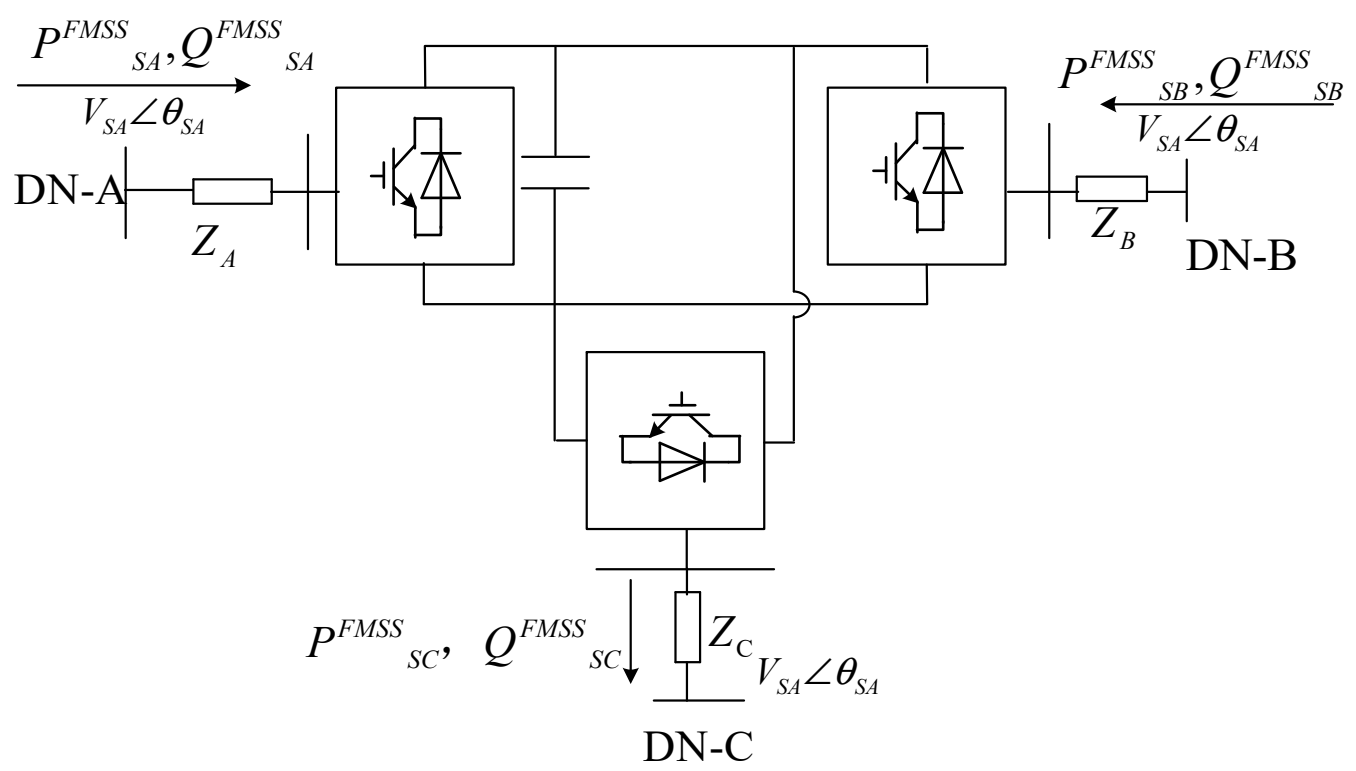

Figure 3. The topology of the three-terminal flexible multi-state switch.

When analyzing the operating constraints of FMSS, only the equality constraints and inequality constraints of the three ports active power and the reactive power of FMSS are considered. When FMSS is running, the active power flows between the three ends of the power grid through the dc side, and the reactive power is only exchanged between this port and the power grid connected with this port. The output range is restricted by the port capacity and the active power of the port. Therefore, the equality constraint is mainly for the coupling relationship between the active power of each port, and the inequality constraint is for the inequality conditions of the active power, reactive power and capacity of each port.

The three-port FMSS can be equivalent to the three-port passive network for analysis. According to the principle of conservation of active power, the equation constraint conditions of FMSS are shown in (14). The inequality constraints of FMSS can be obtained by defining active power, reactive power, apparent power and capacity, as shown in (15).

$$
\begin{gathered}
\left\{\begin{array}{c}
P_{S A}^{\mathrm{FMSS}}+P_{S B}^{\mathrm{FMSS}}+P_{S C}^{\mathrm{FMSS}}+P_{\text {loss }}^{\mathrm{FMSS}}=0 \\
P_{\mathrm{losS}}^{\mathrm{FMSS}}=\frac{k^{\circ} \%}{100}\left[\left(S_{S A}^{\mathrm{FMSS}}\right)^{2}+\left(S_{S B}^{\mathrm{FMSS}}\right)^{2}+\left(S_{S C}^{\mathrm{FMSS}}\right)^{2}\right]
\end{array}\right. \\
\left\{\begin{array}{l}
-\sqrt{\left(S_{A}^{\mathrm{FMSS}}\right)^{2}-\left(P_{S A}^{\mathrm{FMSS}}\right)^{2}} \leq Q_{S A}^{\mathrm{FMSS}} \leq+\sqrt{\left(S_{A}^{\mathrm{FMSS}}\right)^{2}-\left(P_{S A}^{\mathrm{FMSS}}\right)^{2}} \\
-\sqrt{\left(S_{B}^{\mathrm{FMSS}}\right)^{2}-\left(P_{S B}^{\mathrm{FMSS}}\right)^{2}} \leq Q_{S B}^{\mathrm{FMSS}} \leq+\sqrt{\left(S_{B}^{\mathrm{FMSS}}\right)^{2}-\left(P_{S B}^{\mathrm{FMSS}}\right)^{2}} \\
-\sqrt{\left(S_{C}^{\mathrm{FMSS}}\right)^{2}-\left(P_{S C}^{\mathrm{FMSS}}\right)^{2}} \leq Q_{S C}^{\mathrm{FMSS}} \leq+\sqrt{\left(S_{C}^{\mathrm{FMSS}}\right)^{2}-\left(P_{S C}^{\mathrm{FMSS}}\right)^{2}}
\end{array}\right.
\end{gathered}
$$

where $P_{S A}^{\mathrm{FMSS}}, P_{S B}^{\mathrm{FMSS}}, P_{S C}^{\mathrm{FMSS}}$ are the active power exchanged between the three ports of the flexible multi-state switch and the distribution system, respectively. $P_{\text {loss }}^{\mathrm{FMSS}}$ is the operating loss of the flexible multi-state switch, $k$ is the loss coefficient, generally 3-5, $Q_{S A}^{\mathrm{FMSS}}, Q_{S B}^{\mathrm{FMSS}}, Q_{S C}^{\mathrm{FMSS}}$ are the reactive power exchanged between the three ports of the flexible multi-state switch and the distribution system, respectively. $S_{S A}^{\mathrm{FMSS}}, S_{S B}^{\mathrm{FMSS}}, S_{S C}^{\mathrm{FMSS}}$ are the instantaneous apparent power at each port of FMSS, respectively. $S_{A}^{\mathrm{FMSS}}, S_{B}^{\mathrm{FMSS}}, S_{C}^{\mathrm{FMSS}}$ are the rated capacity of the three ports of FMSS, respectively. 


\subsubsection{System Operating Constraints}

The system operating constraints mainly include the system operating voltage level constraints, branch capacity constraints and the system power flow constraints. The system operating voltage horizontal constraint and branch capacity constraint are inequality constraints, as shown in (16) and (17) respectively. The system power flow constraint is an equation constraint, as shown in (18) and (20).

$$
\begin{aligned}
& \left(U_{n}^{\min }\right)^{2} \leq\left(U_{t, n}\right)^{2} \leq\left(U_{n}^{\max }\right)^{2} \\
& 0 \leq\left(I_{t, n m}\right)^{2} \leq\left(I_{n m}^{\max }\right)^{2} \\
& \left\{\begin{array}{c}
\sum_{l \in \psi_{i}} P_{t, n l}=\sum_{m \in \phi_{i}}\left[P_{t, m n}-R_{m n}\left(I_{t, m n}\right)^{2}\right]+P_{t, n} \\
\sum_{l \in \psi_{i}} Q_{t, n l}=\sum_{m \in \phi_{i}}\left[Q_{t, m n}-X_{m n}\left(I_{t, m n}\right)^{2}\right]+Q_{t, n}
\end{array}\right. \\
& \left\{\begin{array}{c}
P_{t, n}=P_{t, n}^{\mathrm{DG}}+P_{t, n}^{\mathrm{FMSS}}-P_{t, n}^{\mathrm{LOAD}} \\
Q_{t, n}=Q_{t, n}^{\mathrm{DG}}+Q_{t, n}^{\mathrm{FMSS}}-Q_{t, n}^{\mathrm{LOAD}}
\end{array}\right. \\
& \left(I_{t, n m}\right)^{2}=\frac{\left(P_{t, n m}\right)^{2}+\left(Q_{t, n m}\right)^{2}}{\left(U_{t, n}\right)^{2}}
\end{aligned}
$$

where $t$ is the network number, $n$ is the number of nodes, $U_{t, n}$ is the voltage of $n$ nodes in the network $t$. In this paper, in addition to the above system constraints, the output range of DG should also be considered. The constraints are shown in (21).

$$
0 \leq P_{D G . i} \leq P_{D G . i \max }
$$

\section{Optimal Algorithm for Maximum Consumption of DG}

In this paper, according to the objective function and constraint conditions described in the previous section, a particle swarm optimization (PSO) algorithm is used to obtain the maximum output scheme of DG under load variations. Due to the large number of control variables, it is easier for the traditional PSO algorithm to get into the local optimal solution. In order to improve the global search ability of PSO in the initial stage and to ensure better search performance in local optimization in the later stages, this study modified the inertia weight of PSO and introduced the second-order nonlinear decreasing inertia weight $w$ [23], so as to better balance the global search ability and local search ability of the algorithm. Compared with the linear decreasing inertia weight, this method has a better ability to get out of the sub-optimal solution. The improved particle swarm updating equation is shown in (22).

$$
\left\{\begin{array}{c}
v_{i d}^{k+1}=w v_{i d}^{k}+c_{1} r_{1}\left(P_{B i d}^{k}-x_{i d}^{k}\right)+c_{2} r_{2}\left(G_{b d}^{k}-x_{i d}^{k}\right) \\
x_{i d}^{k+1}=x_{i d}^{k}+v_{i d}^{k+1} \\
w^{k+1}=w_{\text {start }}-\left(w_{\text {start }}-w_{\text {end }}\right)\left(\frac{k}{T}\right)^{2}
\end{array}\right.
$$

where: $V_{i d}$ is the velocity of the particle; $K$ is the number of current iterations; $w$ is the inertia weight coefficient, which represents the coefficient that the particle maintains its original velocity; $c_{1}$ and $c_{2}$ are the learning factors of particles, respectively self-cognition and social cognition. $P_{i d}$ is the historical optimal value searched by the $i$ th particle itself. $X_{i d}$ is the current position of the particle; $r_{1}$ and $r_{2}$ are random numbers in the interval $[0,1] . P_{g d}$ is the optimal solution currently searched by all particles. Further, $w_{\text {start }}$ is the initial inertia weight; $w_{\text {end }}$ is the inertia weight when iteration reaches the maximum number; $T_{\max }$ is the maximum number of iterations.

The inertia weight of the improved PSO algorithm decreases with the increase of the iteration number, and the change of the initial inertia weight $w_{\text {start }}$ to the end of the iteration weight $w_{\text {end }}$ changes 
with the change of an iteration number as a quadratic function. At the beginning of iteration, the inertia weight is larger, the step size of searching for the optimal particle iteration is larger, and the ability of searching in a wide range is stronger. As the number of iterations increases, the inertia weight decreases, and the step size of the particle iteration to find the optimal one decreases. Therefore, the particles conduct deep excavation near the current optimal solution. The flow chart of the improved PSO algorithm is shown in Figure 4.

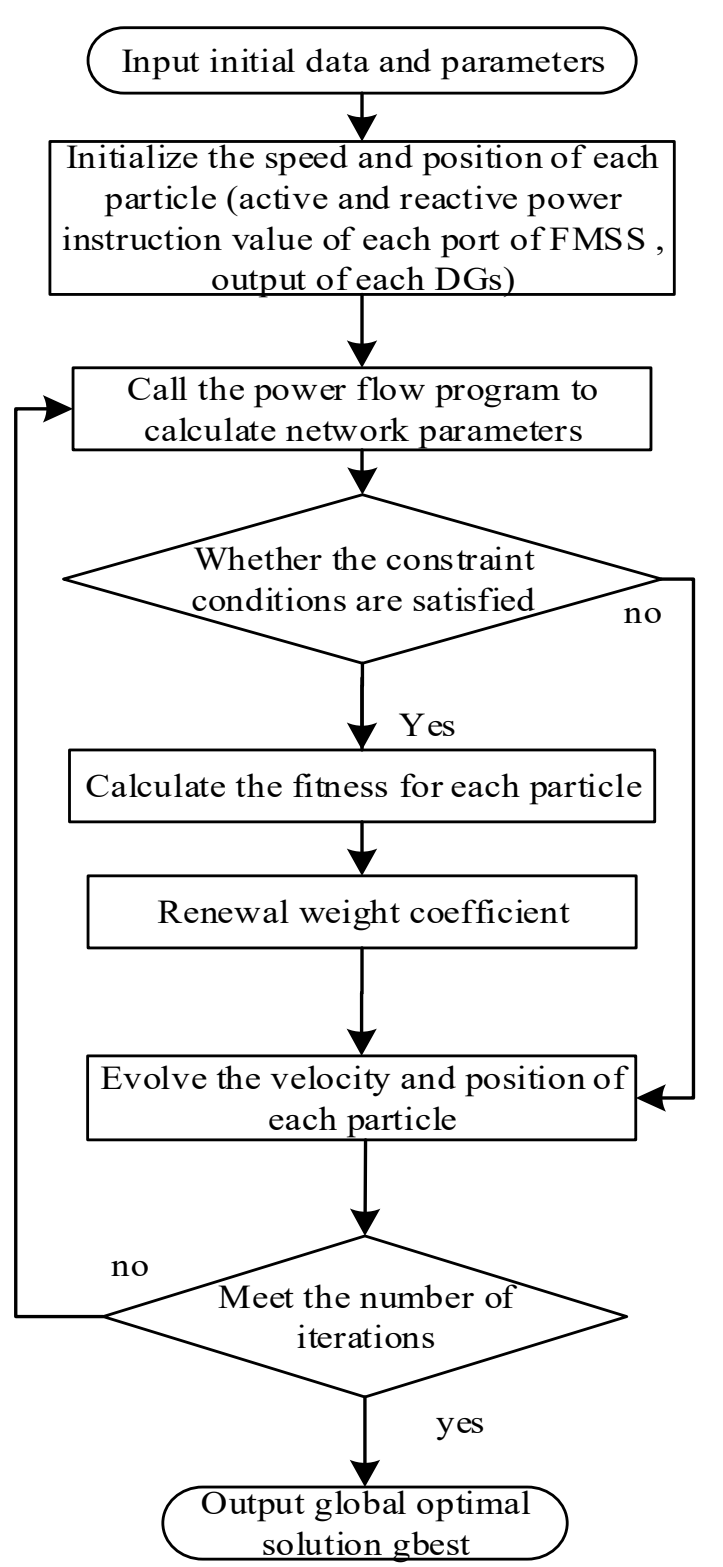

Figure 4. Flow chart of improved particle swarm optimization.

The weight coefficient is determined by combining the analytic hierarchy process and entropy weight method mentioned in Section 2. The specific steps are as follows:

(1) Using the weight coefficient $\omega_{j}$ obtained by the analytic hierarchy process to construct a single objective function, the fitness function of PSO is used to conduct an iterative solution of PSO;

(2) The optimal position reached by each particle (including the local optimal solution and the global optimal solution) is recorded, as are the four single objective function values which correspond to the optimal position of each particle as the basic data; 
(3) The use of basic data on the entropy weight of each objective function value calculation, and according to (13), the comprehensive consideration of the analytic hierarchy process and entropy weight method of each objective function weight coefficient $\lambda_{1}, \lambda_{2}$ through the integration of the two methods obtains the objective function index weight coefficient as $\lambda_{1}=0.734, \lambda_{2}=0.266$.

The weight coefficient is substituted into Equation (12) and is taken as the objective function, and then solved by PSO algorithm to obtain the final optimization result.

\section{Example Simulation}

\subsection{Simulation of Consumption Capacity of Traditional Distribution Network}

In this paper, the ability of the distribution network to consume DG without FMSS is simulated. The IEEE 33-node system is used to access DG at the 17th node. When the network load remains unchanged, the output of the DG is gradually increased from zero to obtain the network node voltage with a different capacity, as shown in Figure 5. In the figure, the $X$-axis is the node number and the $Y$-axis is the per-unit value of node voltage. As shown in the figure, the simulation results show that increasing the output of the distributed power supply leads to increasing node voltage, which may further lead to over-limit voltage. It is shown that the maximum capacity of DG without FMSS access is determined by the system voltage constraint.

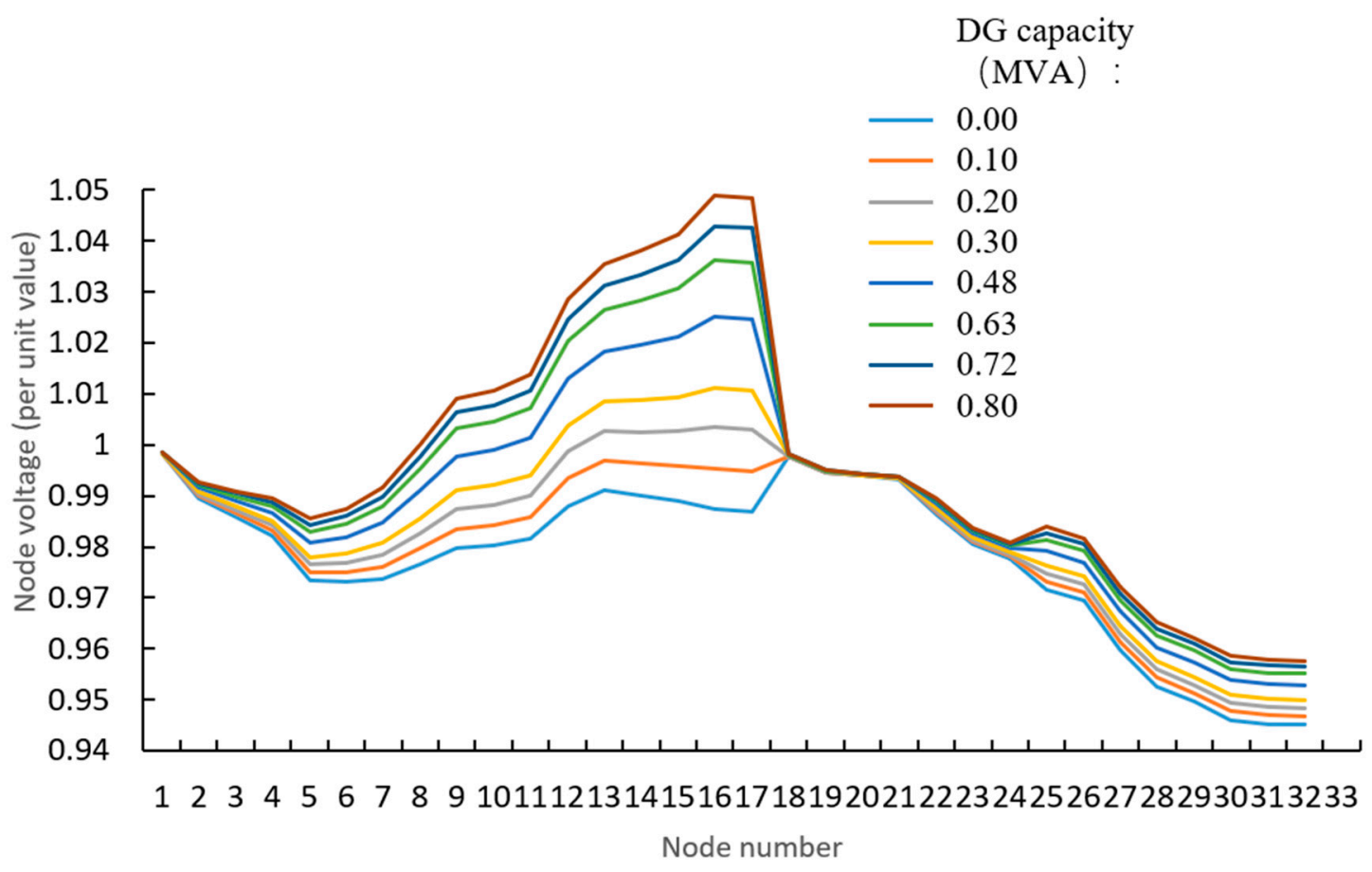

Figure 5. Voltage variation in Network 2 under different outputs of DG.

\subsection{Simulation of Consumption Capacity of Distribution Network with FMSS}

It is proposed that the regulation strategy in this paper is more universal, and especially suitable for complex distribution systems. In this paper, MATLAB is used to establish a distribution network system with three-terminal FMSS, as shown in Figure 6. The three-terminal FMSS connects three IEEE 33-node systems, and FMSS is connected to the distribution network at the 13th node. Several DG are connected in network 1 and network 2 respectively, and their access location and maximum access capacity are shown in Table 1. The distribution network system model established is shown in Figure 6. The access location and the maximum output power of DG are shown in Table 1. In order to simplify the optimization process, only the active power output of DG is considered in this paper. 


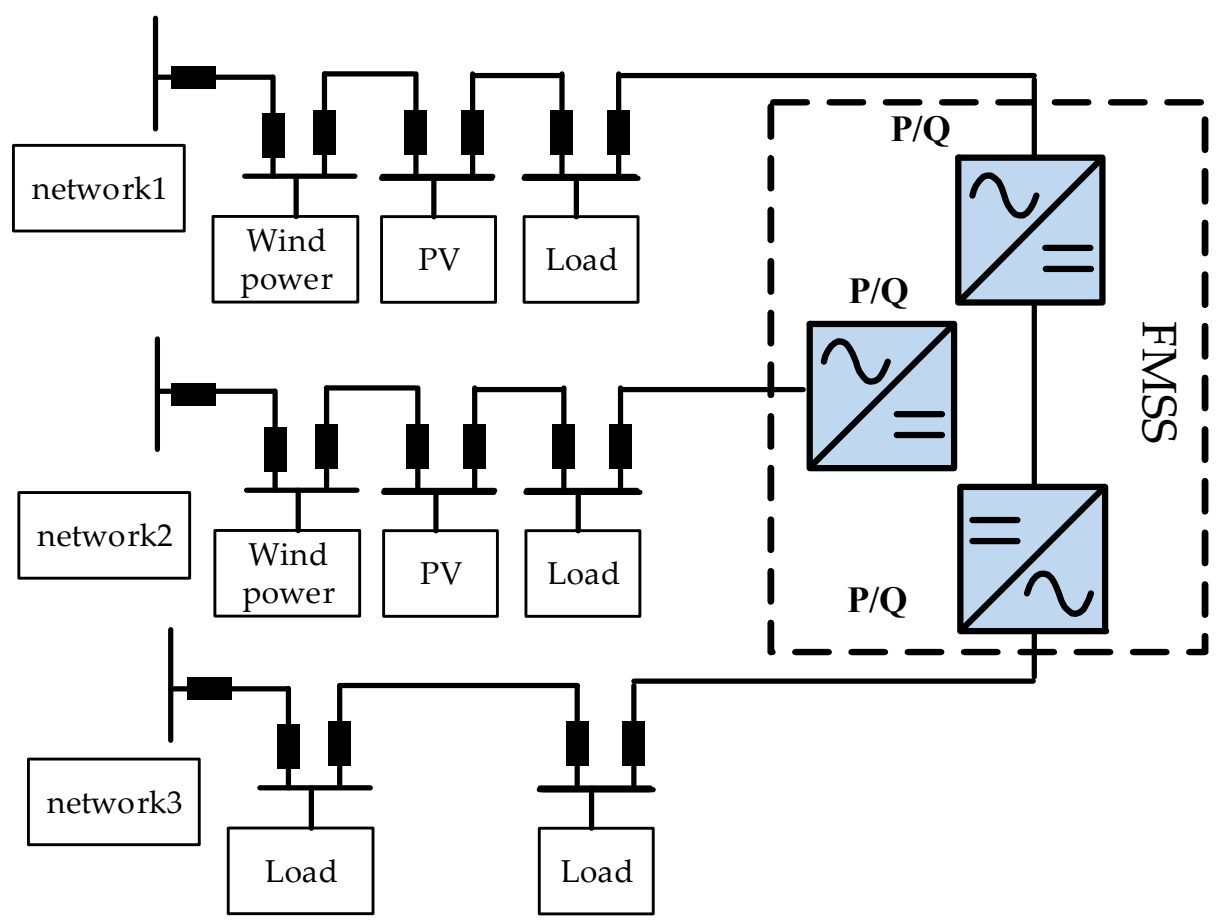

Figure 6. A scenario of the distribution network with three-terminal flexible multi-state switches.

Table 1. Access location and rated power of DG.

\begin{tabular}{cccc}
\hline Network & Node Number & DG Type & The Largest Output \\
\hline 1 & 9 & Wind power & $1.0 \mathrm{MVA}$ \\
1 & 14 & PV & $1.2 \mathrm{MVA}$ \\
1 & 17 & PV & $1.2 \mathrm{MVA}$ \\
\hline 2 & 10 & Wind power & $1.0 \mathrm{MVA}$ \\
2 & 13 & $\mathrm{PV}$ & $1.2 \mathrm{MVA}$ \\
2 & 16 & $\mathrm{PV}$ & $1.2 \mathrm{MVA}$ \\
\hline
\end{tabular}

When studying the maximum consumption of DG, this paper analyzes the operation mode that is most unfavorable to the consumption of DG. According to the operating mode characteristics of the system, when the system operates in the mode of minimum load, the larger the output of DG is, the more limited the operating parameters of the system can be, such as the node voltage, branch power flow, etc. Therefore, it is reasonable to analyze the consumption capacity of DG in the distribution network under the mode of minimum load.

\section{Results and Discussions}

\subsection{Verification of DG Absorption Strategy Based on FMSS}

Under the operating condition of the distribution network system mentioned above, according to the above algorithm, the consumption of DG is adjusted and controlled in real time through the control strategy of FMSS. I added a sequential optimization step in MATLAB program to change the output of photovoltaic power generation (PV) and wind power output throughout the period, means that the load changes the output every half hour for a time node, and results in sequential optimization. The diurnal variation curves of PV, wind power generation and the load are obtained when FMSS is not connected and the rated capacity of different FMSS is connected, as shown in Figure 7. The maximum output of wind power is set as 1 (P.U.) and the maximum output of PV is 1.2 (P.U.). The $Y$-axis represents the output of DG/load capacity, and the X-axis is the time. 
Figure $7 \mathrm{a}$ shows the condition where the distributed power supply is not connected, and Figure $7 \mathrm{~b}-\mathrm{e}$ shows the rated capacity of the FMSS that is connected is 1 MVA,3 MVA,5 MVA and 10 MVA, respectively.

By comparing the output of DG without access to FMSS as shown in Figure 7a and the access to FMSS as shown in Figure $7 \mathrm{~b}-\mathrm{d}$, it can be seen that under the same load change and after FMSS is connected, the output of PV and wind power are significantly improved. In other words, FMSS in the optimization model mentioned above can effectively improve the consumption capacity of the distributed power supply in the distribution network.

As can be seen from Figure $7 \mathrm{~b}-\mathrm{d}$, with the increase of the rated capacity of FMSS, the output of photovoltaic and wind distributed power supply also increases. This indicates that, within a certain range, appropriately increasing the rated capacity of FMSS can increase its capacity to consume DG. Comparing Figure 7d,e, it can be seen that the rated capacity of FMSS has been doubled, but the output of DG has hardly changed. This is because when the FMSS of 5 MVA is connected, the output of DG has been optimized near its rated capacity in accordance with the optimization strategy in this paper. Therefore, it is of little significance to increase the capacity of FMSS in this case.

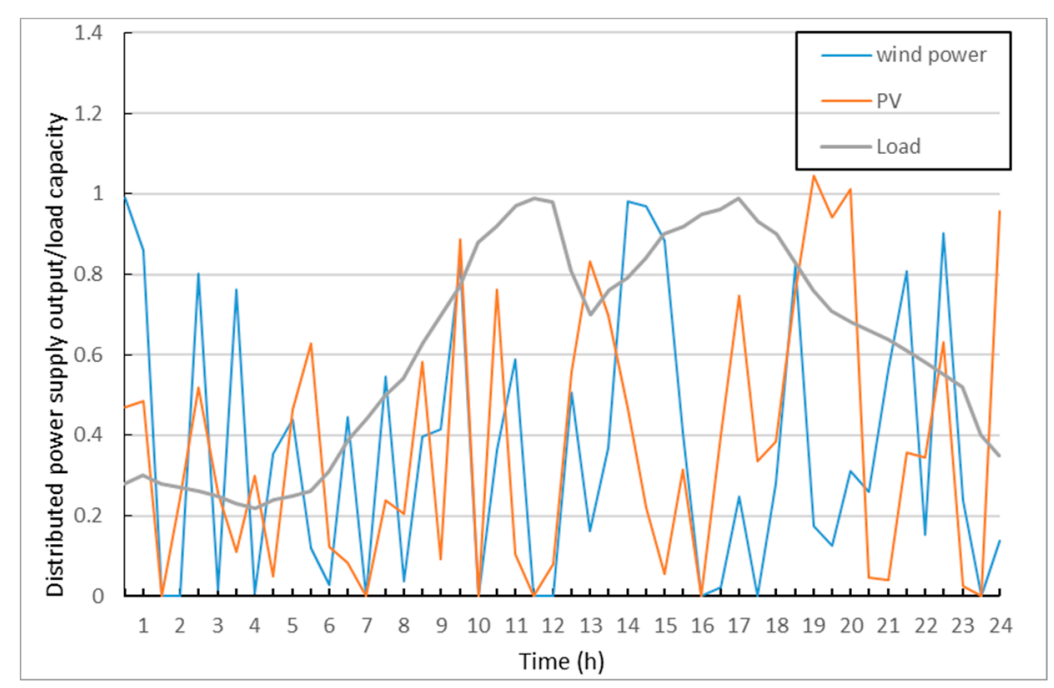

(a) Without flexible multi-state switch (FMSS)

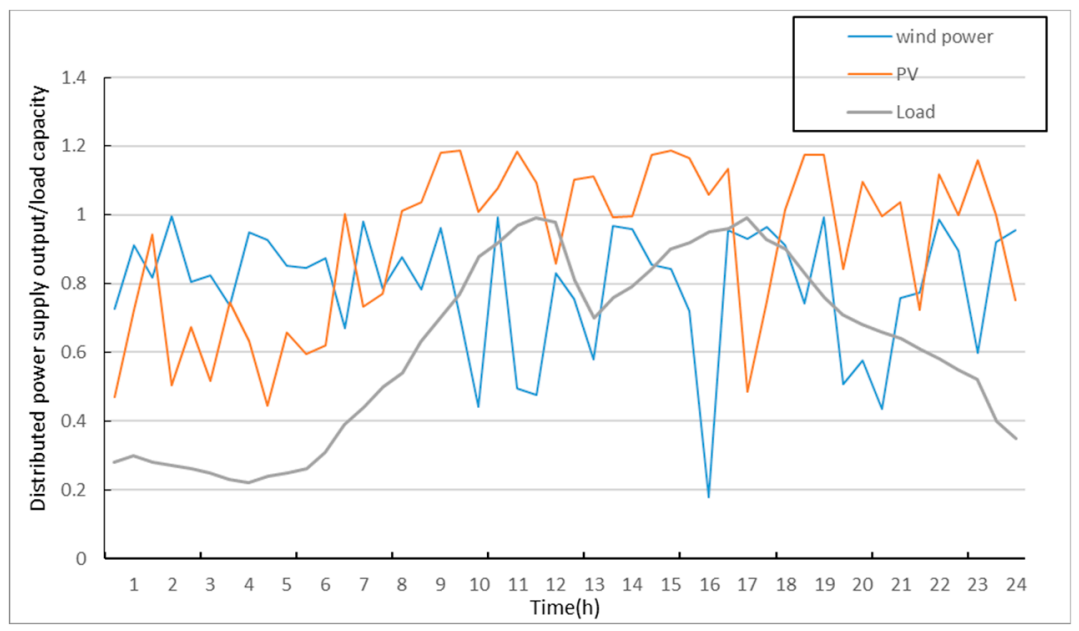

(b) FMSS capacity 1 MVA

Figure 7. Cont. 


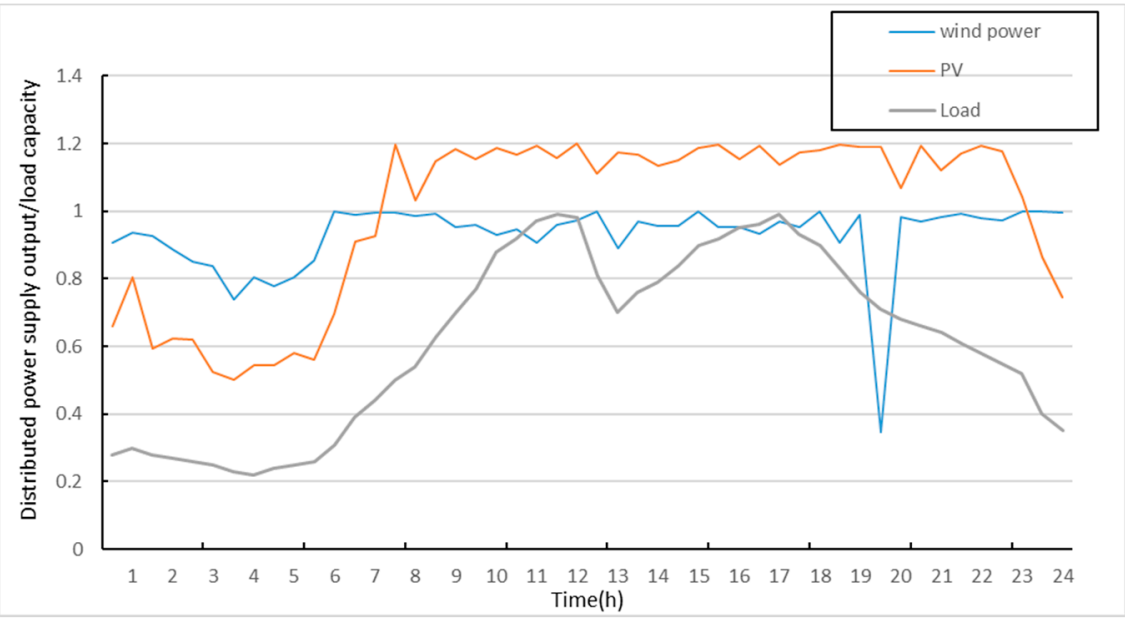

(c) FMSS capacity 3 MVA

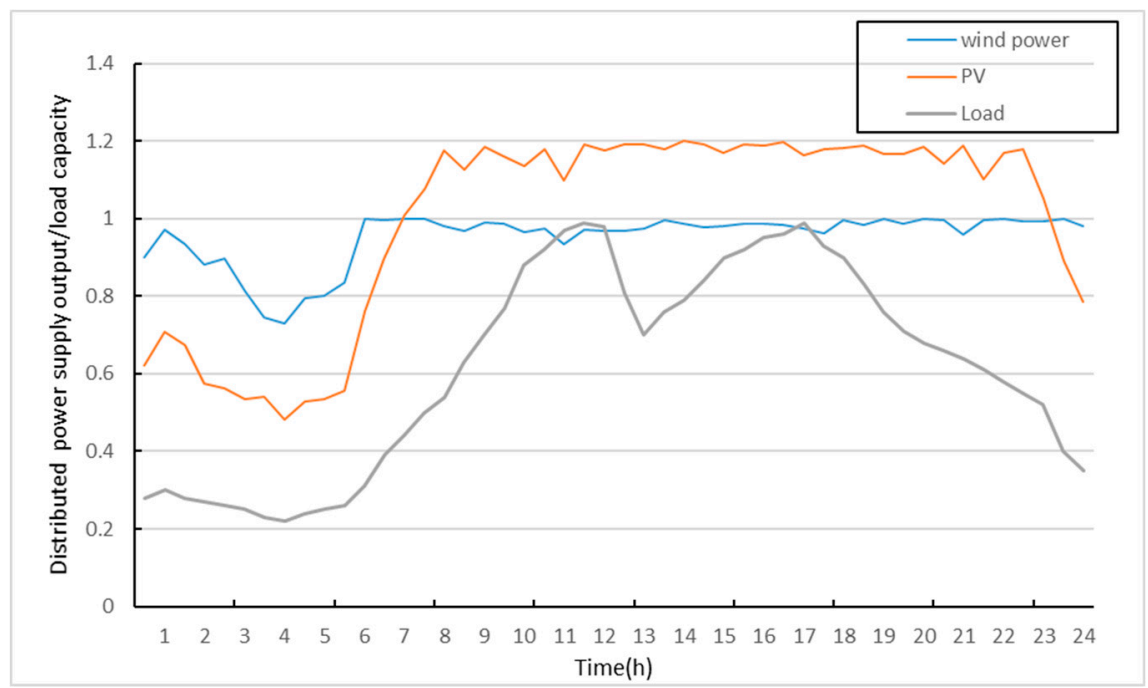

(d) FMSS capacity 5 MVA

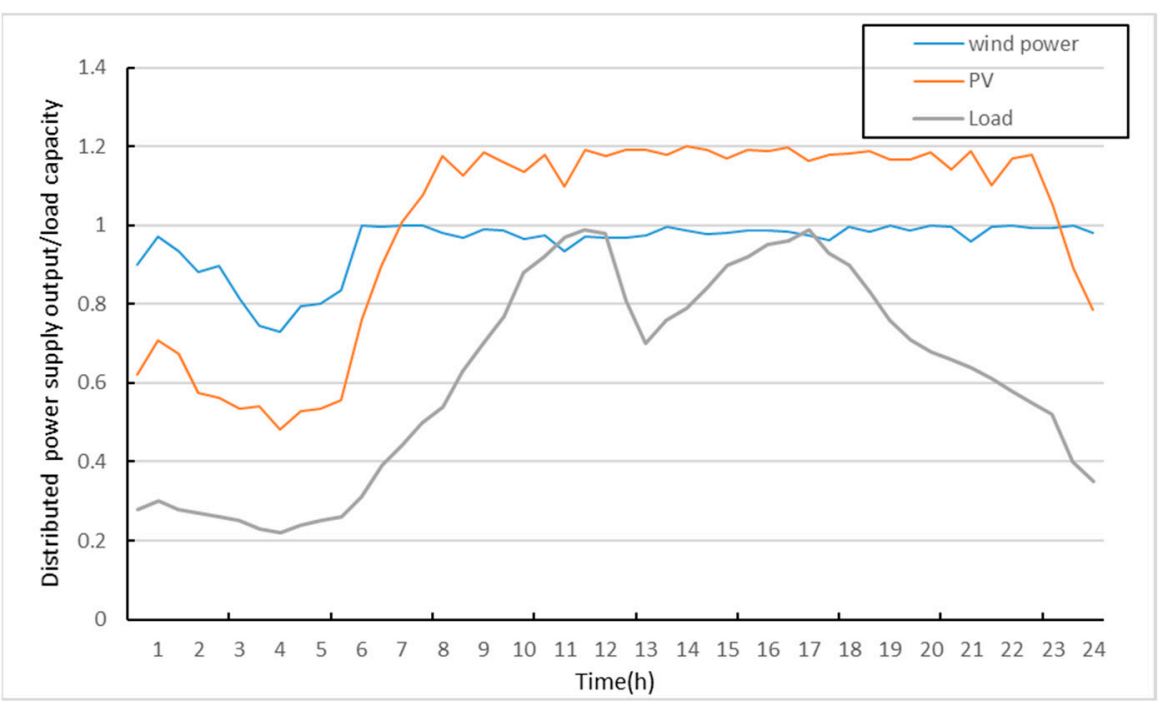

(e) FMSS capacity 10 MVA

Figure 7. Optimal output of DG in network 2 with different FMSS capacity. 


\subsection{Verification of Voltage Fluctuation Suppression Effect of Distribution Network}

Figure 8 shows the voltage changes of each node of network 3 at different time periods throughout the day before and after FMSS is connected. Figure 8a shows the situation when FMSS is not connected, and Figure $8 \mathrm{~b}$ shows the situation when FMSS is connected. Where the $X$-axis represents the time, the $Y$-axis represents the node number, and the $Z$-axis represents the per-unit value of node voltage:

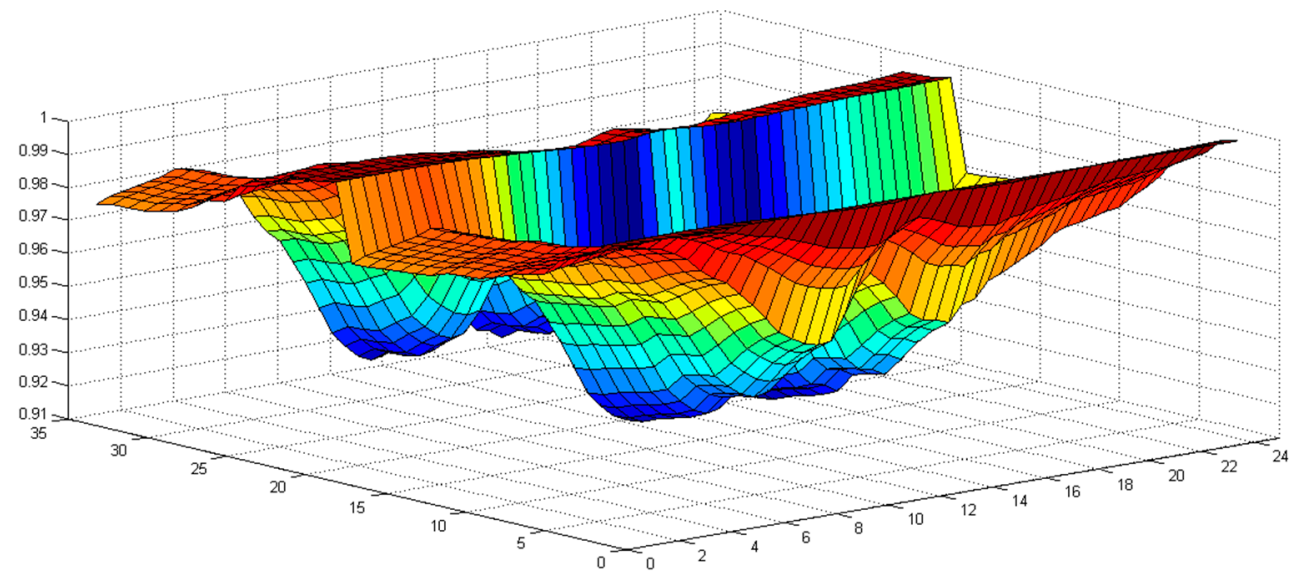

(a) Without FMSS

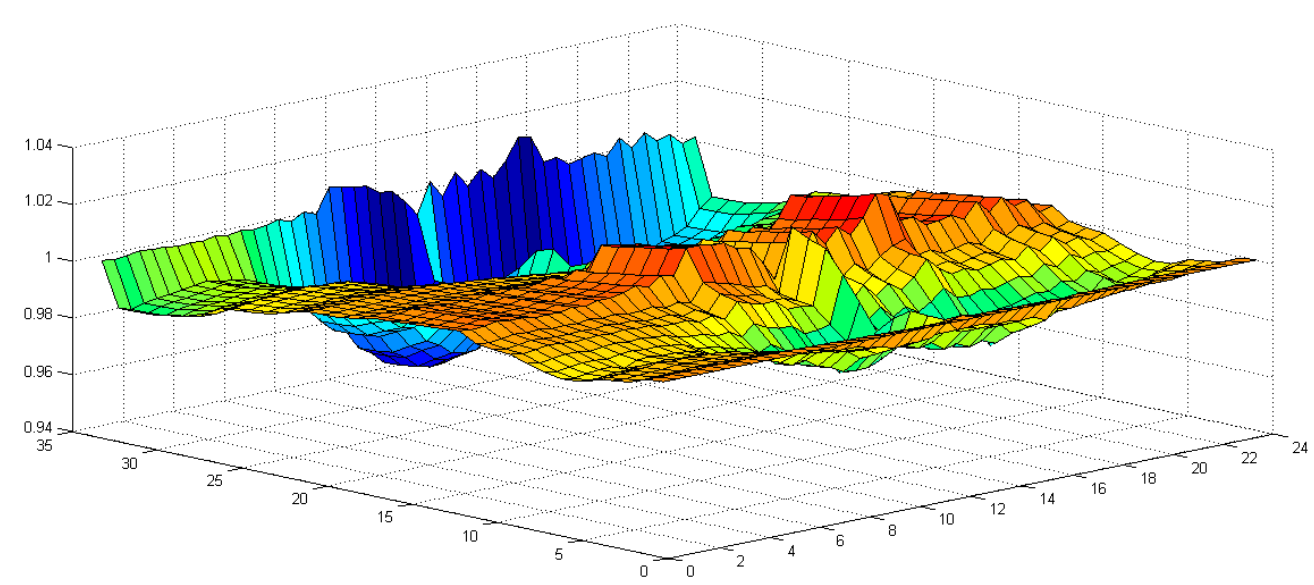

(b) Access FMSS

Figure 8. The node voltage variation of network 3 before and after access FMSS.

The simulation results show that the voltage fluctuation of the distribution network has a gentle approach when FMSS switch is connected. It is shown that FMSS can increase the consumption capacity of the distribution network to DG by regulating the active power flow between the feeders and the reactive power output from ports. At the same time, the feeder load balance is achieved and the node voltage offset is reduced as a whole.

When the capacity of FMSS is 3 MVA, the active and reactive power instructions of each port in the optimization process are shown in Figure 9 and the ordinate unit is MW/MVar. P1, P2 and P3 respectively represent the active output values of three ports of FMSS. Q1, Q2 and Q3 respectively represent the reactive output values of three ports of FMSS. Among them, the active and reactive power instruction values of each port for the first 4 times are shown in Table 2 


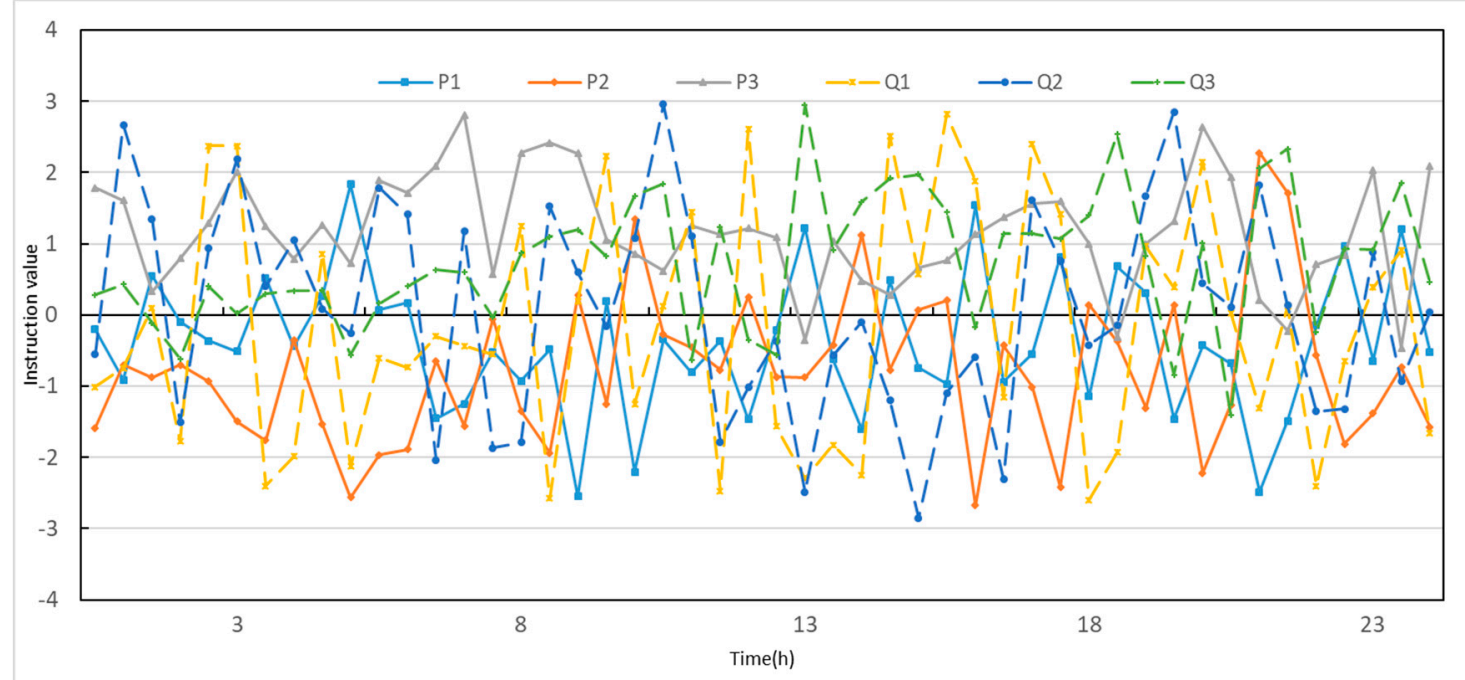

Figure 9. Flexible multi-state switch instructions in the optimization process.

Table 2. Active and reactive command value of each port in the first $4 \mathrm{~h}$.

\begin{tabular}{ccccccccc}
\hline Time & $\mathbf{0 . 5}$ & $\mathbf{1}$ & $\mathbf{1 . 5}$ & $\mathbf{2}$ & $\mathbf{2 . 5}$ & $\mathbf{3}$ & $\mathbf{3 . 5}$ & $\mathbf{4}$ \\
\hline P1 & -0.20057 & -0.90772 & 0.54854 & -0.09768 & -0.36662 & -0.51317 & 0.521791 & -0.43873 \\
P2 & -1.58659 & -0.70286 & -0.87732 & -0.70019 & -0.9288 & -1.49431 & -1.76383 & -0.34716 \\
P3 & 1.78716 & 1.610584 & 0.32878 & 0.797874 & 1.295425 & 2.007476 & 1.242042 & 0.785884 \\
\hline Q1 & -1.01279 & -0.74397 & 0.103522 & -1.77163 & 2.377847 & 2.373122 & -2.39969 & -1.98806 \\
Q2 & -0.55298 & 2.66953 & 1.353455 & -1.50465 & 0.944353 & 2.185228 & 0.399828 & 1.047951 \\
Q3 & 0.279531 & 0.427288 & -0.1135 & -0.61834 & 0.402047 & 0.019273 & 0.296871 & 0.341887 \\
\hline
\end{tabular}

Although seemingly complicated, the combination of the load variation and the DG output of the real time curve in Figure 7, indicates that a complex distribution network system with a varying load can optimize the DG output under this strategy by stable and as large as possible real-time instructions.

Therefore, this algorithm has a good real-time performance. It can adjust the power of FMSS by tracking the change of the load in real time during the system operation to improve the consumption of DG. Meanwhile, FMSS can adjust the active power flow and reactive power flow. This is also the advantage compared to traditional flexible power devices, such as the static var compensator (SVC) which can only regulate the reactive power flow of the line.

\subsection{Comparison with Traditional Reactive Power Compensation Device}

Figure 10 shows the effect of SVC, a traditional reactive power compensation device, used for the consumption of DG. The rated capacity of SVC is 5 MVA. It adopts the same calculation example and optimization algorithm as the FMSS. The $X$-axis represents time and the $Y$-axis is the output of DG or load values. By contrasting Figures 10 and 7d, the same capacity of SVC compared with FMSS can be seen. The former significantly improves the consumption capacity of DG less than the latter. It has been proved that under the condition of the same real-time optimization strategy, FMSS has obvious advantages over SVC because it can change both the active power flow and reactive power flow of the distribution network. 


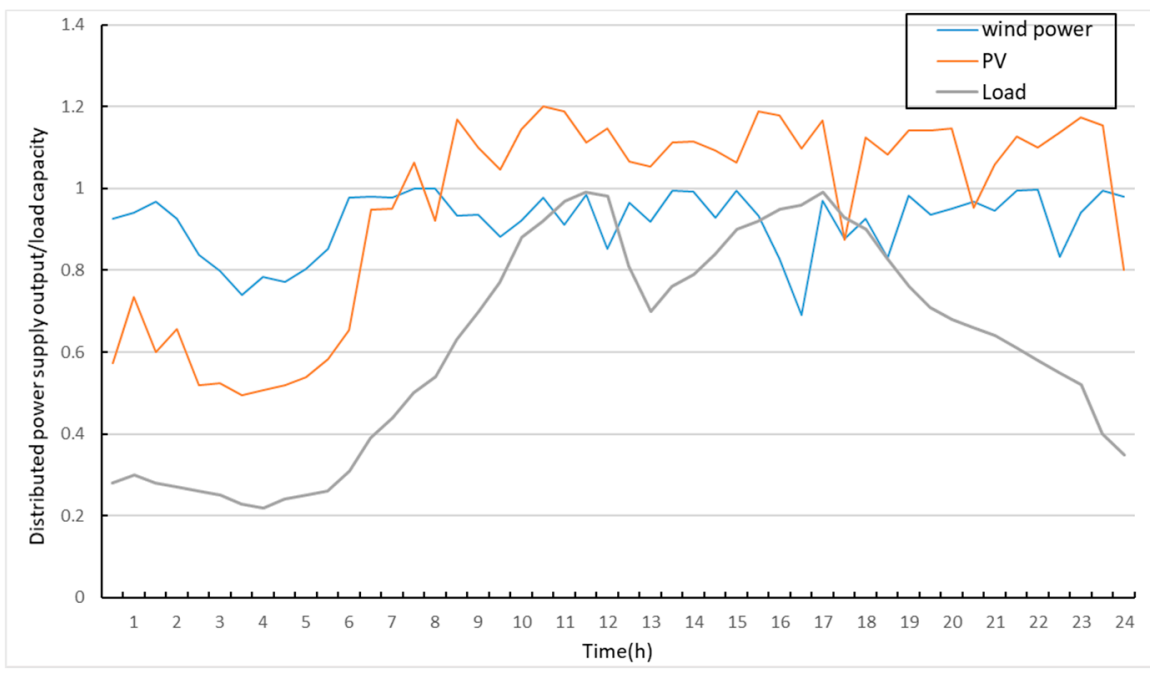

Figure 10. Optimal output of DG in network 2 with static var compensator (SVC).

\section{Conclusions}

This paper focuses on the problem of improving the consumption ability of DG in the distribution network, and proposes a control strategy based on FMSS for maximum consumption of DG in the complex distribution network. Further, improved particle swarm optimization algorithms are used to realize the real-time control of DG.

The effectiveness of this strategy is verified by numerical examples, and the advantages of this strategy are proven by comparison with traditional reactive power compensation devices. Meanwhile, different from most studies based on the planning level, this paper focuses on the real-time operation level of the system and has the advantage of real-time adjustment.

From summarizing the theoretical analysis, the model establishment and the verification of simulation examples in this paper, the following conclusions can be drawn:

1. The main limiting factor of the consumption capacity of DG of the distribution network is the voltage deviation of network lines. FMSS can reduce the voltage deviation of the line by adjusting the active and reactive power flow distribution of the network, thus promoting the consumption ability of DG of the distribution network.

2. By comparing with traditional reactive power compensation devices, such as SVC and STATCOM, it can be seen that FMSS can simultaneously affect active and reactive power flows and have a stronger ability to consume DG.

3. In a certain range, appropriately increasing the rated capacity of FMSS can improve the consumption capacity of DG.

4. In the more complex distribution system, a regulation strategy based on FMSS proposed in this paper, which is oriented to the maximum absorption of the distributed power supply, can dynamically adjust with the change of the system state and has good real-time regulation characteristics. This improves the economy and efficiency of the distribution system.

Author Contributions: The authors contribute as follows: Conceptualization, G.Z.; methodology, G.Z., J.L. and S.L.; software, S.L. and X.Z.; validation, G.Z., S.L. and X.L.; formal analysis, J.L. and S.L.; investigation and resources, J.L. and Y.L.; data curation, S.L.; writing-original draft preparation, G.Z., J.L. and S.L.; writing一review and editing, J.L.; supervision, G.Z., J.L. and Y.L.; project administration, J.L. and Y.L.; funding, J.L. and Y.L.

Funding: This research was funded by National Key R\&D Program of China, 2017YFB0903100 and Science and Technology Projects of State Grid Corporation of China, 521104170043.

Conflicts of Interest: The authors declare no conflicts of interest. 


\section{References}

1. Mahmud, N.; Zahedi, A. Review of control strategies for voltage regulation of the smart distribution network with high penetration of renewable distributed generation. Renew. Sustain. Energy Rev. 2016, 64, 582-595. [CrossRef]

2. Qu, H.; LI, X.; Yang, L.; Huang, Y.; Wang, M.; Huang, J. Multi-objective distribution network dynamic reconfiguration and DG control considering time variation of load and DG. High. Volt. Eng. 2019, 45, 873-881.

3. Razavi, S.E.; Rahimi, E.; Javadi, M.S.; Nezhad, A.E.; Lotfi, M.; Shafie-khah, M.; Catalão, J.P. Impact of distributed generation on protection and voltage regulation of distribution systems: A review. Renew. Sustain. Energy Rev. 2019, 105, 157-167. [CrossRef]

4. Badran, O.; Mekhilef, S.; Mokhlis, H.; Dahalan, W. Optimal reconfiguration of distribution system connected with distributed generations: A review of different methodologies. Renew. Sustain. Energy Rev. 2017, 73, 854-867. [CrossRef]

5. Siyu, G.; Wei, W.; Yuanfu, X.U. Reconfiguration of distribution network for the maximum consumption of distributed generations. Proc. CSU-EPSA 2017, 29, 7-11.

6. Napis, N.; Khatib, T.; Hassan, E.; Sulaima, M. An Improved Method for Reconfiguring and Optimizing Electrical Active Distribution Network Using Evolutionary Particle Swarm Optimization. Appl. Sci. 2018, 8, 804. [CrossRef]

7. Ling, X.F. Optimizing scheduling of active distribution network with flexible DC devices. Electr. Power Constr. 2016, 37, 41-49.

8. Xu, X.; Xu, Z.; Lyu, X.; Li, J. Optimal SVC placement for Maximizing Photovoltaic Hosting Capacity in Distribution Network. IFAC PapersOnLine 2018, 51, 356-361. [CrossRef]

9. Ahmadnia, S.; Tafehi, E. Comparison of Optimum Wind-Solar DG, Statcom and Capacitor Placement and Sizing Based on Voltage Stability Margin Enhancement in Microgrid with Three Different Evolutionary Algorithms. Iran. J. Sci. Technol. Trans. Electr. Eng. 2017, 41, 241-253. [CrossRef]

10. Dong, X.; Liu, Z.; Li, P.; Song, G.; Wu, Z.; Chen, L. Intelligent distribution network control technology based on multi-terminal flexible distribution switch. Proc. CSEE 2018, 38, 86-92.

11. Bloemink, J.M.; Green, T.C. Increasing distributed generation penetration using soft normally-open points. In Proceedings of the IEEE PES General Meeting, Providence, RI, USA, 25-29 July 2010.

12. Qi, Q.; Wu, J. Increasing distributed generation penetration using network reconfiguration and soft open points. Energy Procedia 2017, 105, 2169-2174. [CrossRef]

13. Abdelrahman, M.A.; Long, C.; Wu, J.; Jenkins, N. Optimal operation of Multi-Terminal Soft Open Point to Increase hosting capacity of distributed generation in medium voltage networks. In Proceedings of the 2018 53rd International Universities Power Engineering Conference (UPEC), Glasgow, UK, 4-7 September 2018; pp. 1-6.

14. Liang, H.S.; Li, S.W.; Bai, L.Q.; Liu, C.; Li, W.; Xu, J.; Wang, Q.B. Calculation of maximum access capacity of distributed power supply in flexible distribution network based on SNOP. Power Constr. 2008, 39, 69-76.

15. Wang, C.; Sun, C.; Li, P.; Wu, J.; Xing, F.; Yu, Y. SNOP-based Operation Optimization and Analysis of Distribution Network. Autom. Electr. Power Syst. 2015, 39, 82-87.

16. Wang Chengshan, S.O.N.G.; Guanyu, L. Ahybrid Optimization Method for Distribution Network Operation with SNOP and Tie Switch. Proc. CSEE 2016, 36, 2315-2321.

17. Ji, H.; Wang, C.; Li, P.; Ding, F.; Wu, J. Robust Operation of Soft Open Points in Active Distribution Networks with High Penetration of Photovoltaic Integration. IEEE Trans. Sustain. Energy 2018, 10, 280-289. [CrossRef]

18. Wang, Q.; Liao, J.; Su, Y.; Lei, C.; Wang, T.; Zhou, N. An optimal reactive power control method for distribution network with soft normally-open points and controlled air-conditioning loads. Int. J. Electr. Power Energy Syst. 2018, 103, 421-430. [CrossRef]

19. Zhang, T.T.; Liu, C.; Gong, J.F.; Zhao, W.X. Research on Distributed Generation Access System in Distributed Grid. Northeast. Electr. Power Technol. 2016, 37, 4-7.

20. Bloemink, J.M.; Green, T.C. Increasing photovoltaic penetration with local energy storage and soft normally-open points. In Proceedings of the 2011 IEEE Power and Energy Society General Meeting, Detroit, MI, USA, 2011; pp. 1-8.

21. Chen, S.J. Study on Distribution Network Optimal Operation and Distributed Generation Hosting Capacity; Tianjin University: Tianjin, China, 2016. 
22. Zeng, D.; Yao, J.; Yang, S.; Wang, K.; Li, Y. Economy comparison of VSC-HVDC with different voltage Levels. Autom. Electr. Power Syst. 2011, 35, 98-102.

23. Sha, Y.; Qiu, X.Y.; Ning, X.J.; Han, X. Multi-Objective Optimization of Active Distribution Network by Coordinating Energy Storage System and Flexible Load. Power Syst. Technol. 2016, 40, 1394-1399. 